\title{
Enquêter dans les lieux d'enfermement. Retour collectif sur une expérience de recherche
}

Bénédicte Michalon, Tristan Bruslé, Olivier Clochard, Mathilde Darley, Olivier Milhaud et Marie Morelle

\section{Q OpenEdition}

\section{Journals}

Édition électronique

URL : https://journals.openedition.org/cdg/7324

DOI : $10.4000 /$ cdg. 7324

ISSN : 2107-7266

Éditeur

UMR 245 - CESSMA

\section{Référence électronique}

Bénédicte Michalon, Tristan Bruslé, Olivier Clochard, Mathilde Darley, Olivier Milhaud et Marie Morelle, "Enquêter dans les lieux d'enfermement. Retour collectif sur une expérience de recherche », Carnets de géographes [En ligne], 15 | 2021, mis en ligne le 30 avril 2021, consulté le 27 mai 2021. URL : http:// journals.openedition.org/cdg/7324; DOI : https://doi.org/10.4000/cdg.7324

Ce document a été généré automatiquement le 27 mai 2021.

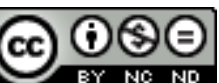

La revue Carnets de géographes est mise à disposition selon les termes de la Licence Creative Commons Attribution - Pas d'Utilisation Commerciale - Pas de Modification 4.0 International. 


\title{
Enquêter dans les lieux d'enfermement. Retour collectif sur une expérience de recherche
}

\author{
Bénédicte Michalon, Tristan Bruslé, Olivier Clochard, Mathilde Darley, \\ Olivier Milhaud et Marie Morelle
}

\section{Introduction}

De 2008 à 2015, l'équipe TerrFerme, composée de chercheur.e.s et ingénieur.e.s en sciences sociales (géographie et science politique), a traité la thématique de l'enfermement à partir de l'étude de différents types de lieux ${ }^{1}$ : prisons, centres de rétention administrative pour étranger.e.s, logements contraints de travailleurs ${ }^{2}$. Les enquêtes ont été menées dans plusieurs pays : l'Allemagne, la Roumanie, la France, l'Espagne ${ }^{3}$, le Qatar et le Cameroun. Partout, le regard s'arrête en premier lieu sur les limites matérielles, les murs, les clôtures, les portes, les grilles et les grillages. Derrière tous ces artefacts de la frontière, de la séparation, du tri et du contrôle, une foule d'« indésirables » (Agier, 2008) est reléguée : des migrants sans papiers, des travailleurs immigrés, des détenus... Dans ces mondes sociaux soustraits aux regards extérieurs évolue une multitude d'acteurs aux motivations et aux trajectoires contrastées: les personnes recluses elles-mêmes, bien sûr, mais aussi les acteurs faisant profession de l'organisation de la réclusion (policiers, surveillants, employeurs, personnel médical) comme de sa contestation ou, a minima, de la défense des droits des personnes recluses (acteurs associatifs, religieux, juridiques). Les membres de notre équipe ont adopté une démarche méthodologique inspirée de l'ethnographie, associant un travail de recueil d'entretiens plus ou moins formalisés, de discussions informelles avec les différents acteur.trices et d'observations des interactions qui se nouent entre eux. Nous nous positionnions ainsi dans la continuité d'un vaste ensemble de recherches en sciences sociales s'appuyant sur des enquêtes qualitatives largement inspirées de l'analyse goffmanienne des «institutions totales " (Goffman, [1961] 1968). Cette dernière a en effet durablement influencé la production de connaissances empiriques sur 
l'enfermement et a largement contribué à légitimer, dans l'arène académique, la conduite d'enquêtes ethnographiques au sein même des institutions fermées. Si les modes d'investigation de la réclusion restent aujourd'hui très diversifiés - travail sur archives, sur plans, analyse et déconstruction de statistiques, de discours publics, etc. (De Galembert, Henneguelle, Touraut, 2017) -, l'enquête par observations et entretiens semble particulièrement adaptée au travail de documentation et d'analyse de l'expérience des sujets (reclus ou membres des différents corps professionnels présents entre les murs) et a ainsi constitué un socle méthodologique commun à l'ensemble de l'équipe. Cette approche des lieux de privation de liberté ou de contrôle des mobilités n'est donc pas à proprement parler inédite; l'originalité de la démarche tient cependant à la réflexion méthodologique engagée collectivement par une équipe pluridisciplinaire et travaillant dans des contextes nationaux et sur des dispositifs de réclusion diversifiés quant à leurs finalités et au degré de contrainte qu'ils imposent aux personnes placées sous leur contrôle.

2 Nous partagions à l'entame du projet une même inquiétude : celle de ne pas pouvoir pénétrer dans les lieux visés par nos enquêtes, caractérisés par certaines formes de clôture vis-à-vis du monde extérieur et donc a priori difficilement accessibles au regard $\mathrm{du}$ chercheur. Tout terrain a, il est vrai, ses formes de fermeture, et toute enquête comporte son lot d'obstacles et de verrous imprévus. Toutefois, la thématisation de l'ouverture/fermeture tient ici une place centrale, tant dans les récurrentes mais vaines tentatives de réforme des politiques d'enfermement, que dans l'élucidation de leurs logiques politiques et sociales (pour une présentation synthétique de cette dialectique voir Darley, Lancelevée, Michalon, 2013 ; Turner, 2016). La mise au point d'un protocole d'enquête ne saurait donc s'en extraire. Les modalités d'accès au terrain et les négociations menées avec les institutions et leurs gardiens pour obtenir le droit d'entrer occupent ainsi une part conséquente des réflexions d'ordre méthodologique sur l'enfermement (Bony, 2015 ; Gill, 2016 ; Hall, 2012 ; Milhaud, 2017 ; Moran, Pallot, Piacentini, 2009 ; Morelle, 2015 ; Pallot, 2007 ; Piacentini, 2004 ; Piacentini, Pallot, Moran, 2009 pour n'en citer que certains).

Tableau 1. Présentation des enquêtes et des terrains

\begin{tabular}{|c|c|c|c|c|}
\hline $\begin{array}{l}\text { Nom du ou de } \\
\text { la chercheur.e }\end{array}$ & Institution & Pays & $\begin{array}{l}\text { Statut annoncé } \\
\text { du ou de la } \\
\text { chercheuse }\end{array}$ & Accès aux lieux \\
\hline Tristan Bruslé & $\begin{array}{lr}\text { Logement } & \text { fermé pour } \\
\text { main } & \text { d'œuvre } \\
\text { étrangère } & \text { (labour camp) }\end{array}$ & Qatar & Chercheur & $\begin{array}{l}\text { Oui (avec l'accord du } \\
\text { contremaître } \\
\text { responsable du camp) }\end{array}$ \\
\hline $\begin{array}{l}\text { Olivier } \\
\text { Clochard }\end{array}$ & $\begin{array}{l}\text { Zone d'attente pour les } \\
\text { personnes en instance } \\
\text { d'éloignement }\end{array}$ & France & $\begin{array}{l}\text { Bénévole de la } \\
\text { Cimade }\end{array}$ & $\begin{array}{ll}\text { Refusé } & \text { comme } \\
\text { chercheur, } & \text { accepté } \\
\text { comme bénévole } & \end{array}$ \\
\hline $\begin{array}{l}\text { Mathilde } \\
\text { Darley }\end{array}$ & $\begin{array}{l}\text { Centre de rétention } \\
\text { administrative pour } \\
\text { étranger.e.s }\end{array}$ & Allemagne & $\begin{array}{l}\text { Aumônière } \\
\text { stagiaire } \\
\text { chercheuse }\end{array}$ & $\begin{array}{l}\text { Oui, mais circulations } \\
\text { internes restreintes }\end{array}$ \\
\hline $\begin{array}{l}\text { Bénédicte } \\
\text { Michalon }\end{array}$ & $\begin{array}{l}\text { Centre de rétention } \\
\text { administrative pour } \\
\text { étranger.e.s }\end{array}$ & Roumanie & Chercheuse & $\begin{array}{l}\text { Oui, mais circulations } \\
\text { internes restreintes }\end{array}$ \\
\hline
\end{tabular}




\begin{tabular}{|l|l|l|l|l|}
\cline { 5 - 5 } & Foyer d'hébergement & & Bénévole & Oui \\
\hline Marie Morelle & Prison & Cameroun & Chercheuse & $\begin{array}{l}\text { Oui, accès illimité dans } \\
\text { un premier temps, accès } \\
\text { interdit dans un second } \\
\text { temps }\end{array}$ \\
\hline Olivier Milhaud & Prison & France & Chercheur & $\begin{array}{l}\text { Oui, mais circulations } \\
\text { internes restreintes }\end{array}$ \\
\hline Djemila Zeneidi & $\begin{array}{l}\text { Logement fermé pour } \\
\text { main d'œuvre } \\
\text { étrangère Espagne }\end{array}$ & Chercheuse & Non \\
\hline
\end{tabular}

3 Sans arguer d'un particularisme méthodologique nécessaire à leur appréhension, force est pourtant de constater que les dynamiques relationnelles au sein des lieux d'enfermement sont souvent à l'origine de conflits émotionnels singuliers chez les chercheur.e.s (Le Caisne, Proteau, 2008 ; Liebling, 2001 ; Wacquant, 2002), qui tentent de les résoudre en négociant une identité acceptable pour leurs différents interlocuteur.trices (Chantraine, 2004; Cliquennois, 2006). Ils sont alors tenus d'endosser certains rôles ou d'accepter ceux qu'ils se voient assigner, qui pourront d'ailleurs changer au cours de l'enquête. La littérature scientifique consacrée à l'expérience de l'enfermement et les théorisations de l'expérience en général invitent ainsi les scientifiques à se livrer à un exercice de réflexivité : l'expérience des sujets enquêtés ne pourrait se comprendre sans que celle des chercheur.e.s ne le soit elle-

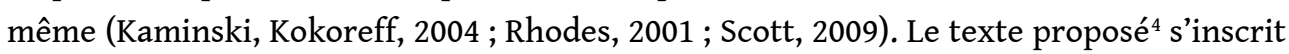
dans ce projet d'explicitation, au moins partielle, de l'expérience de recherche et de retour critique sur une démarche scientifique de mise en dialogue d'enquêtes menées en parallèle sur un objet d'étude commun. Nous nous concentrerons sur l'étape de l'accès aux enquêté.e.s et sur les différents types de négociation menées pour " entrer " sur le terrain ainsi que sur leurs effets dans la conduite des enquêtes. Cette étape nous semble particulièrement révélatrice de deux évolutions contemporaines des dispositifs de contrainte étudiés. D'abord, enquêter entre les murs est possible grâce au mouvement d'ouverture relative des institutions de réclusion : les négociations menées avec les autorités publiques (première partie) et avec des acteurs associatifs (deuxième partie) ont ouvert à certain-es d'entre nous les portes des lieux visés et ont largement conditionné nos positions intramuros. Toutefois, les difficultés rencontrées par d'autres ont montré les limites de ce mouvement (troisième partie) et nous ont poussé.e.s à enquêter également hors les murs (quatrième partie) : c'est ici l'organisation de la surveillance en un continuum social et spatial (Foucault, 1975 ; Wacquant, 2001) qui a guidé nos enquêtes. Chacun des groupes d'acteur.trices avec lesquels nous avons dû négocier pour enquêter représente à la fois des "portiers » (Olivier de Sardan, 1995, p. 19) avec lesquels il a fallu composer, et une première source d'information et de compréhension de l'objet d'étude. Notre réflexion collective montre donc, à partir d'une entrée méthodologique, que la "normalisation» (Snacken, 2002) relative de l'enfermement s'inscrit dans un mouvement plus large consacrant des modalités plus diffuses de surveillance, moins scrutées et contrôlées par des observateurs extérieurs. 


\section{Enquêter intramuros : les chercheur.e.s face aux institutions de contrôle des lieux d'enfermement ${ }^{5}$}

4 L'enquête est, "entre les murs" comme au sein d'autres lieux d'investigation, tributaire des conditions d'accès, lesquelles sont ici marquées par une fermeture plus ou moins nette et par le contrôle exercé sur les lieux par une institution, publique ou privée. Notre méthodologie s'est donc construite dans un rapport à une autorité donnée, qu'elle ait été garante de notre accès à certains types d'espaces ou qu'à l'inverse, elle nous ait contraint.e.s à des solutions de repli dont nous questionnons les conséquences sur le choix des lieux et modalités d'enquête.

\section{Accéder aux lieux : négociations et objectivation du contrôle}

5 L'accès aux lieux d'enfermement nécessite généralement l'obtention d'une accréditation préalable auprès des autorités qui gèrent et contrôlent les établissements visés.

6 Nos demandes d'accès, lorsqu'elles ont été officiellement adressées aux institutions, ont été formulées au titre de "chercheur.e " et motivées par le souhait de rencontrer les personnes enfermées et les autres acteur.trices de l'enfermement. Elles ont reçu des réponses très diverses de la part des institutions compétentes, suivant des temporalités variables (de quelques heures à une année pleine). Il est par ailleurs apparu que l'accès aux établissements pouvait différer entre différents lieux placés sous une même autorité (les prisons sous la responsabilité de l'Administration pénitentiaire en France par exemple) ou entre pays, qu'ils émanent ou non d'un même ensemble régional (centres de rétention de l'Union européenne par exemple). Surtout, le degré d'ouverture/fermeture des lieux, matérialisé par les murs et autres dispositifs de contrainte, ne s'est pas systématiquement reflété dans les dispositions de l'institution à accueillir des chercheur.e.s en son sein. La posture à l'égard de nos demandes a donc souvent été teintée de méfiance, en raison du potentiel critique associé aux recherches sur l'enfermement. La présentation de soi et de son travail (intentions, objectifs, techniques d'enquête) était alors déterminante dans l'«examen d'entrée » auquel plusieurs d'entre nous se sont trouvés soumis. La mise en avant de l'appartenance au monde académique, et parfois d'étranger.e au contexte national, a pu ici jouer, notamment dans les contextes européens d'enquête, en faveur de la crédibilisation de l'étude effectuée, et limiter ainsi les effets de domination des «imposants" (Chamboredon et al., 1994; Laurens, 2007). Ailleurs, ils ont à l'inverse accru les difficultés. La crainte qu'un jugement de valeur soit porté sur des institutions du Sud, souvent perçues comme dysfonctionnelles par les bailleurs du Nord, et dans certains contextes autoritaires dans lesquels les prisons sont des espaces de détention politique, illustrent la portée de la situation géographique et de l'inscription dans le jeu géopolitique et post-colonial des lieux d'enquête.

7 Ce processus de négociation, et notamment la demande formulée par certains représentants institutionnels de disposer de la grille d'entretien préalablement à toute rencontre, a cependant été à la source de nombreux dilemmes, entre honnêteté et contraintes pressenties : jusqu'où dévoiler nos intentions? Il a fallu, sur chacun des terrains, trouver le mode de présentation le plus favorable à l'obtention des accréditations. Certain.e.s d'entre nous ont mis en avant leur intérêt pour 
l'architecture, l'organisation des lieux, à l'instar de l'enquête dans les prisons françaises et les centres de rétention allemands, en passant sous silence la dimension de contrôle et des rapports de pouvoir inhérents à toute spatialisation des phénomènes sociaux. D'autres ont dû taire leur préoccupation pour la matérialisation du contrôle et insister au contraire sur leur intérêt pour les relations sociales internes, l'entrée par l'espace s'avérant parfois beaucoup moins "rassurante " pour l'institution que nous avions pu naïvement le penser avant d'aller sur le terrain - c'était notamment le cas pour l'enquête dans les centres de rétention roumains. Il est surtout apparu que les points sensibles de la recherche variaient grandement d'une institution et d'un contexte à l'autre, illustrant la capacité qu'ont les acteur.trices institutionnels à problématiser de manière réflexive et critique leur institution. Ainsi, si l'appartenance à une équipe dont le financement public a pu être perçu comme une forme de caution dans le contexte français et européen, le rapprochement thématique de lieux apparemment éloignés dans leur conception a en revanche donné lieu à des interprétations contradictoires de la part de nos interlocuteur.trices institutionnels. Parfois considéré comme un décentrement bienvenu du regard (notamment par les acteurs du Nord ou de l'Ouest de l'Europe présumant souvent que la comparaison avec le Sud ou l'Est leur serait favorable), il risquait également de nous fermer certaines portes. Comment justifier en effet, auprès des employeurs et employeuses de maind'œuvre étrangère, le rapprochement opéré entre logements de main d'œuvre $^{6}$ et prisons sans mettre en péril nos demandes d'accès? Dans la plupart des pays étudiés, notre statut d'étranger.e semble cependant avoir contribué à limiter la méfiance : notre position apparaissait alors extérieure, d'une part, aux débats politiques locaux et, d'autre part, aux relations souvent ambiguës et de surveillance mutuelle entre organisations non gouvernementales (ONG) et directions des établissements de réclusion.

8 Les procédures suivies pour accéder à nos différents lieux d'enquête illustrent les lenteurs, les obstacles et les formes possibles d'arbitraire instaurés par les administrations, et ce tant dans des systèmes autoritaires que démocratiques; le cas français le montre de manière particulièrement nette. En effet, les prisons relèvent en France d'une administration d'État assurant un service public (à la différence des entreprises employant des étranger.e.s en Espagne ou au Qatar). L'Administration pénitentiaire française a par ailleurs l'habitude d'agréer la conduite d'enquêtes scientifiques entre ses murs (à la différence des autres institutions de réclusion incluses dans notre réflexion) et compte même en son sein un département "recherche", partenaire du milieu universitaire. Olivier Milhaud s'est cependant trouvé confronté à un ensemble de procédures astreignantes pour pouvoir mener son étude sur le système pénitentiaire français. Il avait pourtant déjà eu accès aux prisons dans le cadre d'un engagement associatif au GENEPI (Groupement étudiant national d'enseignement aux personnes incarcérées), qu'il avait définitivement interrompu au moment des terrains par peur de mal négocier l'articulation entre recherche et militantisme. À l'époque de son engagement associatif, l'accès aux lieux avait nécessité un entretien de moralité au commissariat de police de son quartier. Pour la recherche en revanche, la lettre de recommandation de son directeur de thèse, avec le sceau du CNRS, devait servir classiquement de caution scientifique, tout comme les grilles d'observation et d'entretien qui l'accompagnaient, tandis que le fait d'insister sur la dimension géographique de la recherche (paysage observé depuis la cellule par exemple) devait en minimiser la dimension potentiellement critique et militante quant aux conditions de 
détention. Suite à diverses relances, le troisième courrier arriva à bon port, fut transmis aux directions régionales des établissements concernés, avant d'arriver sur le bureau des chefs d'établissement. Certains ne répondirent jamais, dont un établissement qui venait de connaître une spectaculaire évasion à l'arme de guerre. D'autres refusèrent. D'autres acceptèrent, avec ou sans condition sur l'usage du dictaphone. Tous les chefs d'établissement souhaitèrent rencontrer Olivier Milhaud avant tout entretien avec les détenus. Il fallut aussi rediscuter de ses hypothèses et de ses connaissances du monde carcéral avec un chef d'établissement et, parallèlement, avec le chef de cabinet du Directeur de l'Administration pénitentiaire à Paris. Il s'agissait chaque fois, pour ses interlocuteurs, de lui faire comprendre qu'étant plus jeune et non membre de l'Administration pénitentiaire, il ne pourrait tirer de son travail qu'une connaissance limitée et très partielle de l'univers carcéral. Ressortait alors de cette tentative de cadrage de la recherche par l'institution la dichotomie " chercheur.e dangereux.se/chercheur.e en danger» (Chantraine, Chauvenet, 2005), qui constituait ipso facto l'Administration pénitentiaire comme protectrice du chercheur ou de la chercheuse autant que de l'ordre public, et comme seule autorité souveraine sur le lieu d'enfermement, auquel le ou la chercheur.e resterait irréductiblement étranger.e.

9 Au final, ce qui a facilité l'accès au terrain de l'institution semble moins être la présentation d'une question de recherche sous un angle aussi "apolitique " que possible que le fait de faire coïncider notre réflexivité avec celle des acteur.trices de l'administration (voir Fassin, 2015 par exemple), dont les points de vue peuvent par ailleurs être hétérogènes.

\section{Contrôle et auto-contrôle dans la collecte des données intramuros}

10 Une fois obtenue l'autorisation d'enquêter entre les murs, nous avons été confronté.e.s à la question de la marge de manœuvre qui nous était autorisée en termes de collecte de données. Cette problématique s'est principalement posée à travers nos circulations dans les lieux d'enquête. Les chercheur.e.s peuvent parfois bénéficier du droit d'y circuler librement (Scheer, 2017), voire se voir confier un jeu de clés (Bosworth, 2017; Earle, 2014 ; Turnbull, 2015), symbole entre tous du pouvoir détenu par les surveillants. Tel n'a pas été le cas pour les membres de notre équipe puisqu'une fois à l'intérieur, à l'exception de Marie Morelle et de Tristan Bruslé, nos déplacements au sein des espaces fermés sont demeurés sous contrainte, oscillant entre lieux possibles et lieux interdits. L'agencement des établissements exerce une influence décisive sur les pratiques d'enquête. Les structures carcérales et de rétention sont caractérisées par la présence d'un personnel de surveillance et d'un outillage technologique destiné au contrôle caméras de vidéosurveillance, portes blindées et fermées à clé, œilletons, etc. (Michalon, 2015; Chantraine, Scheer, Milhaud, 2012). Les chercheur.e.s ne peuvent passer outre. Ils et elles se voient imposer un circuit, parfois présenté comme indispensable à leur propre sécurité. Selon le mode d'accès aux lieux, il nous a fallu apprendre à nous plier aux logiques de découpage de l'espace. Les déplacements des membres de l'équipe ayant pu enquêter intramuros en tant que chercheur.e.s ont été différents de ceux des collègues ayant collaboré avec des acteurs non gouvernementaux : ces derniers n'ont souvent qu'un accès partiel (voire pas d'accès du tout) aux espaces dévolus aux "gardiens », ce qui complique d'autant l'observation de leurs pratiques de contrôle. En tout état de cause, la surveillance de nos modalités de 
déplacement entre les murs s'est révélée être pour les institutions un moyen de contrôler la collecte des données.

À ce premier mode de restriction de l'accès aux informations ont été ajoutées des contraintes techniques. Ainsi, pour Bénédicte Michalon, les autorisations d'accès dans deux centres de rétention en Roumanie ont été assorties de l'interdiction stricte d'utiliser tout matériel d'enregistrement sonore et de prise de vue, contrainte également imposée aux étrangers retenus. Cette interdiction d'enregistrer et de photographier a été rencontrée par tous les membres de l'équipe autorisés à enquêter intramuros (sauf Tristan Bruslé), ce qui a rendu impossible la collecte de certaines informations. Une fois sur place, la circulation de Bénédicte Michalon dans les lieux a été fortement encadrée. Elle a néanmoins effectué ses entretiens sans sélection préalable des interlocuteurs par la direction des centres. Dans l'un d'eux, elle les a réalisés dans une salle de réunion du personnel sans dispositif spécifique de surveillance, mais opportunément placée entre le bureau du directeur et celui de la psychologue. Dans l'autre, en revanche, elle a été installée dans la salle de visite (le " parloir ») avec demande expresse de la part des policiers d'en laisser la porte ouverte, " pour sa sécurité ». Elle a rapidement constaté qu'une caméra de vidéosurveillance enregistrait tout ce qui se passait dans cette pièce. Ainsi, tout en répondant à l'injonction à la transparence qui prévaut dans l'espace européen (Darley, Lancelevée, 2016 ; Fischer, 2015), l'institution peut poser des limites claires à la collecte des données, entraînant des formes d'auto-censure chez les chercheur.e.s.

11 L'enquête de Marie Morelle dans la prison centrale de Yaoundé est révélatrice de ces enjeux d'autocensure et d'inconfort émotionnel qui limitent les investigations entre les murs. Elle a obtenu sans difficulté, en 2010, une autorisation du Secrétaire d'État à l'Administration pénitentiaire à enquêter et circuler librement dans la prison, des bureaux de l'administration aux quartiers de détention. Se retrouver au milieu des quartiers surpeuplés dits quartiers Kosovo ${ }^{7}$, abritant chacun en moyenne 1300 détenus ${ }^{8}$, ou au milieu de ceux réservés aux personnes souffrant de maladies mentales, constitue cependant une épreuve, tant physique que morale. Les flux incessants, le bruit sourd, les cris, les bousculades et les empoignades sont venus exacerber le caractère anxiogène et étouffant de la prison, et plus encore de ces quartiers, malgré l'effort de Marie Morelle pour mettre à distance les discours des gardiens et ses propres représentations sur la dangerosité des prisonniers. De plus, être parfois la seule femme au centre des regards de cet univers masculin a pu accroitre son inconfort, voire susciter chez elle un sentiment de vulnérabilité. Ces désagréments l'ont poussée à chercher des solutions de repli et à mener son enquête dans le quartier le moins peuplé : le quartier des condamnés à mort, occupé par 22 prisonniers. Cette solution de «ménagement » de soi, plus que nécessaire au regard des spécificités de l'enquête, a pu l'orienter vers certains prisonniers plus que vers d'autres et la conduire à minorer les observations dans d'autres espaces de la prison - biais potentiel qu'elle a tenté de corriger en fréquentant régulièrement d'autres prisonniers et d'autres zones de l'établissement petit à petit.

Les contraintes qui pèsent sur la vie dans les lieux d'enfermement génèrent enfin des formes plurielles d'autocensure chez les personnes enfermées, ce dont les enquêtes scientifiques doivent tenir compte. La prison et le centre de rétention sont les lieux d'une importante production discursive de la part des personnes recluses, amenées à s'exprimer sur leur situation ou retracer leur parcours, que ce soit devant leurs 
avocat.es, des défenseur.seuses militant.es, ou le personnel qui les prend en charge. Cette production discursive est cruciale car elle peut conditionner la remise en liberté, le maintien derrière les barreaux ou l'expulsion des étranger.e.s. Elle peut également influer sur les conditions de vie intramuros, par exemple si la personne enfermée arrive à s'attirer les faveurs du personnel. Le contexte de réclusion incite donc (voire contraint) à la production de récits standardisés, visant à mettre en avant la condition de victime du locuteur et, ainsi, à contester la légitimité de l'enfermement (Darley, 2010), en censurant au contraire d'autres aspects du parcours. Dans un contexte de formatage institutionnel très fort des récits, les chercheur.e.s sont alors confronté.e.s à la difficulté de faire émerger d'autres types de discours.

12 Les enquêtes que certain.e.s membres de l'équipe ont pu mener révèlent toutes les ambivalences des institutions face aux regards extérieurs portés sur elles. $\mathrm{Si}$, dans le contexte européen, elles se prêtent dans une certaine mesure au jeu de la transparence exigé par le discours sur la "normalisation» de la réclusion, elles ne manquent pas d'imposer aux chercheur.e.s des restrictions à la collecte des données et entraînent ainsi des pratiques d'auto-censure, aussi bien chez les enquêteurs.rice.s que chez les enquêté.e.s. Par ailleurs, même lorsque les portes de la prison s'ouvrent à la recherche, la brutalité de la vie entre les murs peut conduire les chercheur.e.s, comme Marie Morelle au Cameroun, à restreindre d'eux-elles-mêmes la liberté d'action dont ils et elles pourraient théoriquement disposer.

\section{Enquêter intramuros : collaborer avec des organisations non gouvernementales et tenir son rôle}

13 Dans certains contextes, il nous est apparu préférable de négocier l'autorisation d'enquêter non pas directement auprès de l'institution étatique en charge des lieux, mais auprès d'acteur.trices non gouvernementaux accrédités à y pénétrer. L'injonction à la transparence des institutions de réclusion et la juridicisation des rapports sociaux (Rostaing, 2009; Fernandez et al., 2015) garantissent en effet désormais à certains intervenant.e.s non gouvernementaux, nationaux ou internationaux - de manière plus ou moins durable et toujours soumise à conditions - l'accès aux prisons et centres de rétention notamment, grâce à un contrat passé avec la direction (policière ou pénitentiaire). Ces acteur.trices représentent ainsi une source d'information importante pour la recherche, tout comme une entrée possible sur le terrain (Corbet, Michalon, 2017). Cette modalité particulière d'enquête est fréquemment utilisée pour la collecte de matériaux ethnographiques dans les lieux d'enfermement des étranger.e.s (Clochard, Fischer, 2007; Darley, 2008; Makaremi, 2010). Elle peut résulter de difficultés à obtenir, auprès des institutions étatiques, une autorisation officielle en tant que chercheur.e, mais aussi d'un choix méthodologique mettant notamment l'accent sur l'expérience de l'enfermement par les populations recluses ou sur les logiques et pratiques professionnelles des intervenant.e.s non-gouvernementaux en milieu fermé.

14 Au sein du projet TerrFerme, la collaboration avec des ONG nous a notamment permis d'accéder aux lieux d'enfermement des étranger.e.s et aux étranger.e.s enfermé.e.s en Roumanie, en Allemagne et en France. Dans ce dernier cas, l'accès aux zones d'attentes ${ }^{9}$ étant subordonné à une autorisation attribuée par la Police aux frontières (PAF), Olivier Clochard a effectué plusieurs demandes écrites pour enquêter en tant que chercheur au 
sein de la zone d'attente de Roissy (ZAPI 3) ${ }^{10}$. Il a également sollicité à plusieurs reprises la direction centrale de la PAF. Dans le même temps, il avait demandé, en tant que bénévole de la Cimade ${ }^{11}$, une carte d'accès aux zones d'attente auprès du ministère de l'Intérieur, afin de suivre et comprendre le rôle des membres de l'association au sein de ces lieux. La PAF a cependant considéré les deux demandes - formulées pour l'une au titre de "chercheur» et pour l'autre sous l'étiquette de "bénévole» - comme incompatibles. Elle a finalement refusé à Olivier Clochard l'accès aux zones d'attente pour ses activités de recherche. Ses visites en zone d'attente se sont donc effectuées exclusivement avec la Cimade, au titre de son activité bénévole.

Les enquêtes de Mathilde Darley dans un centre de rétention allemand et de Bénédicte Michalon avec des étrangers sortis de rétention en Roumanie illustrent également l'importance des coopérations pouvant exister, en matière de défense des droits des étranger.e.s, entre sphère académique et arène associative. Les intervenant.e.s non étatiques étaient en effet les seuls acteurs non policiers présents dans les centres de rétention visés par les enquêtes. Ils étaient donc aisément identifiables et leur fonction d'assistance en rétention était reconnue tant par les autorités nationales, qui leur accordaient le droit d'entrer dans les centres, que par leurs homologues des autres pays européens et les étranger.e.s en besoin d'assistance. Les aumônier.e.s suivi.e.s par Mathilde Darley ont une vocation pastorale explicite, cependant largement outrepassée dans leurs pratiques quotidiennes d'assistance, qui incluent également une aide juridique et sociale aux étranger.e.s (Darley, 2014). L'ONG approchée par Bénédicte Michalon a certes été créée par une congrégation religieuse mais elle centre ses activités sur l'assistance juridique et sociale en rétention et à la sortie. Les négociations avec les acteur.trices non étatiques ont donc dû tenir compte de ces spécificités, qui ont largement déterminé les positions de bénévoles occupées par Mathilde Darley et Bénédicte Michalon. Expliquant leur activité de recherche et les enjeux du travail mené, elles ont émis le souhait d'enquêter à l'intérieur même des centres, aux côtés des acteur.trices non gouvernementaux. Leurs interlocuteur.trices ont cependant répondu de manière différente à leurs demandes : il a été proposé à Mathilde Darley d'endosser le statut d'aumônière stagiaire pour accompagner quotidiennement les intervenant.e.s religieux.euses en rétention. Si Bénédicte Michalon n'a pas pu entrer en rétention par le biais de l'ONG contactée, celle-ci lui a en revanche permis de rencontrer des personnes sorties de rétention et soutenues, voire hébergées, par l'organisation - nous y reviendrons. Ses activités de bénévolat, connues des responsables policiers, ont agi auprès d'eux comme une sorte de "garantie " morale lui permettant en parallèle d'entrer en rétention pour y réaliser des entretiens dans le cadre de sa recherche alors que ces mêmes activités et l'engagement militant et contestataire prêté aux acteurs associatifs présents entre les murs auraient sans doute, dans d'autres contextes, justifié un refus d'autorisation.

Entrer sur le terrain par une collaboration avec l'ONG autorisée à intervenir sur place nous a donc ouvert un espace d'observation relativement large, profitant des marges de manœuvre déjà négociées par les ONG dans leur pratique quotidienne des lieux d'enfermement des étranger.e.s. Les membres de l'équipe qui ont été bénévoles pour des acteurs non étatiques ont pu inscrire leur présence sur le terrain dans le cadre des relations privilégiées établies de longue date entre le monde académique et la sphère militante (Fassin, 2005). Cette proximité place cependant le chercheur dans une position d'équilibriste, entre engagement et distance critique vis-à-vis de son objet et de son terrain de recherche (D'Halluin, 2005). Il est en effet associé aux discours et à 
l'image portés par l'association qui l'autorise, sous couvert de bénévolat, à enquêter auprès des personnes recluses. L'entrée par la voie non gouvernementale a pu nous désigner comme étant un.e de ses membres. Cette désignation légitimait dès lors « naturellement » un rôle de défenseur des droits des « enfermé.e.s » et par conséquent a pu avoir des incidences sur le déroulement des enquêtes, notamment à travers des jeux d'assignation dont la justification relevait le plus souvent de questions de sécurité (Liebling, 2001).

17 Le fait d'endosser le rôle d'intervenant.e non gouvernemental.e rend généralement l'intérêt pour «l'autre versant» (policier, judiciaire, etc.) peu légitime aux yeux des acteur.trices non gouvernementaux auxquels le ou la chercheur.e s'identifie ou est identifié. La préservation de sa place au sein du dispositif passe donc par l'intégration des codes et valeurs plus ou moins explicitement édictés par celles et ceux qu'il ou elle accompagne et qui impliquent généralement le maintien ostensible d'une distance visà-vis des agent.es du contrôle. Mais le risque est alors de rendre compte prioritairement de la parole des personnes enfermées concentrant l'action associative en raison de leur condition de "victimes» (de persécutions, de violences, d'injustice, etc.). Le confort offert par cette représentation quasi manichéenne des rapports de pouvoir permet au scientifique de donner à son intérêt souvent prioritaire (voire exclusif) pour les personnes soumises à l'enfermement une légitimation morale ou éthique (au détriment des professionnel.les). En outre, le fait de devoir l'accès au terrain à la bienveillance d'acteur.trices associatifs rend également délicate l'étude de leurs pratiques et de leurs discours. Ayant généralement justifié son entrée sur le terrain par l'intérêt porté à celles et ceux qui subissent la contrainte de la réclusion, le ou la chercheur.e peut craindre d'apparaitre déloyal.e s'il ou elle élargit son champ d'analyse aux pratiques des acteur.trices institutionnels ou associatifs qui lui ont ouvert les portes du lieu fermé (Massicard, 2002). Ce fut notamment le cas au cours de l'enquête de Mathilde Darley dans un centre de rétention allemand: initialement motivée par l'intérêt porté aux expériences des étranger.e.s enfermé.e.s, celle-ci a finalement largement porté sur les pratiques professionnelles de ces acteurs.rices singulier.e.s de la rétention que représentaient les aumônier.e.s assumant le rôle de conseillers juridiques. Cet angle privilégié, qui a guidé l'essentiel des publications tirées de l'enquête, n'a pourtant jamais été assumée face aux enquêté.e.s eux-mêmes, plaçant la chercheuse dans un conflit de loyauté fort à leur égard: ces dernier.e.s voyaient en effet dans les motivations de la recherche les mêmes ressorts que ceux qui dictaient leur engagement quotidien en rétention et conféraient alors au discours scientifique, par la « reconnaissance savante » dont il était censé être auréolé (Massicard, 2002), un pouvoir stratégique de mise en visibilité et de légitimation de leur action de défense des étranger.e.s. Se conformer au rôle attendu signifiait notamment réprimer les questions ou les tentatives d'observations qui auraient traduit une curiosité jugée incongrue au regard de la fonction officiellement endossée dans l'enceinte du lieu. Sa position de stagiaire auprès d'un pasteur l'a par ailleurs conduite à être très vite identifiée comme " alliée » des intervenant.e.s religieux et "adversaire » des membres du personnel de police, dans un contexte de tensions croissantes entre ces deux familles d'acteur.trices au sein de l'arène rétentionnaire. Après la fin de son "stage ", la poursuite de l'enquête par des entretiens avec des policiers en poste en rétention a donc avant tout nécessité une forme de distanciation (temporelle notamment) avec le rôle qui lui avait d'abord été assigné. 
Les enquêtes conduites intramuros par une collaboration avec une ONG rappellent la portée heuristique des échanges entre la sphère académique et le milieu associatif. Elles rappellent aussi et surtout combien les chercheur.e.s sont tributaires des formes d'ouverture de l'institution aux acteur.trices non gouvernementaux, et de leur capacité à identifier et tenir les rôles attendus d'eux de la part des différents groupes en présence (Le Caisne, 2017 ; Rhodes, 2001 ; Scheer, 2017).

\section{Enquêter sans autorisation : l'intervention des personnes enfermées}

19 Lorsque les portes des institutions refusent de s'ouvrir et que les possibilités de mener une recherche embarquée via les acteurs non étatiques sont inexistantes, il reste alors la possibilité de faire sans les autorités. Plusieurs d'entre nous ont procédé de la sorte. Ils et elles s'étaient en effet heurté.e.s à des demandes restées sans suite ou à des refus fermes des autorités en charge des lieux visés et ont donc choisi de faire fi de l'aval de celles-ci, d'adapter leurs enquêtes aux contingences du terrain et d'improviser pour contourner les interdictions d'accès. De manière intéressante, ces situations ont principalement concerné les études consacrées aux formes privées de logement contraint, l'une au Qatar, l'autre en Espagne, organisées par les employeurs pour leur main d'œuvre étrangère. Dans les deux cas, il ne s'agissait pas d'institutions d'enfermement stricto sensu mais de dispositifs de confinement (Akoka, Clochard, 2015) fondés sur la captivité et la relégation socio-spatiale. Ces deux enquêtes ont ainsi révélé avec force d'une part la méfiance partagée par les acteurs privés vis-à-vis du travail de recherche, d'autre part leur exemption de l'injonction à la transparence faite aux institutions publiques. Les contraintes rencontrées et les démarches déployées par les chercheur.e.s ont ainsi éclairé le poids des enjeux propres à des secteurs économiques stratégiques, tout comme la crainte des acteurs privés que ne soient publicisées les conditions de travail et de logement des ouvrier.e.s étranger.e.s, points de mire des médias internationaux.

20 L'enquête a pu démarrer, dans le cas de Tristan Bruslé, grâce au soutien voire à la complicité des enquêtés confinés, en l'occurrence des ouvriers. Son entrée dans les camps de travailleurs népalais au Qatar s'est faite en premier lieu hors des lieux d'habitation, principalement avec des responsables associatifs, eux-mêmes en relation avec ces travailleurs peu qualifiés. Il a pu sans trop d'encombres visiter certains lieux durant une heure ou deux et parler avec les habitants car il était toujours accompagné de Népalais qui résidaient eux-mêmes dans les camps ou y avaient des amis. Il a néanmoins ressenti une forte suspicion à son égard et rencontré beaucoup de difficultés pour prendre contact avec les responsables des entreprises employant les ouvriers et les gestionnaires des camps. Il a donc décidé d'entrer et de séjourner dans un camp où il disposait de contacts anciens, après avoir demandé une autorisation officieuse au responsable népalais du camp, intermédiaire entre les travailleurs et l'entreprise et qui n'y résidait plus lui-même. Il l'autorisa, sans l'accord de l'employeur, à y rester quelques nuits. Avec l'aide d'ouvriers, rencontrés pour certains lors de précédents séjours, il s'installa dans une pièce inoccupée. Suivant les conseils des habitants, en conflit larvé avec l'employeur, et désirant mener à bien sa recherche, il dépassa finalement le délai initialement imparti tout en gardant en permanence à 
l'esprit la crainte de se faire expulser du camp, voire du pays, et la mise en danger de ceux qui avaient facilité son séjour.

21 Face à l'impossibilité d'accéder aux foyers et logements des saisonnières migrantes, il n'y a pas eu, pour Djemila Zeneidi, d'autre alternative que de mener l'enquête hors les murs. Malgré de nombreuses demandes auprès d'employeurs et de la mairie de Cartaya en Espagne, en charge du dispositif d'encadrement des saisonnières agricoles marocaines, elle n'a pas été autorisée à accéder à leurs lieux d'habitation. Ces dernières font en effet l'objet d'un encadrement serré où la surveillance et le contrôle s'exercent tout au long du séjour, dans le cadre d'une prise en charge planifiée par les services municipaux. Seules lui ont été proposées des visites organisées dans des foyers et exploitations "modèles", visites réservées aux journalistes et autres visiteurs extérieurs. Quant aux employeurs, ils ont refusé de manière nette l'accès à leurs exploitations. Tout comme la mairie, ils justifiaient leur refus par la méfiance qu'ils nourrissaient à l'égard des universitaires, assimilés ici aux journalistes ayant dénoncé les conditions de vie et de travail de leurs employées. Cette impossibilité d'accès aux lieux-clés que représentaient les hébergements et les exploitations l'a conduite à envisager une solution de repli : mener l'enquête dans la rue et dans les commerces où les ouvrières venaient faire leurs achats. Celles-ci habitaient cependant assez loin du centre-ville et ne disposaient que d'un temps libre limité. De plus, elles ne parlaient pas sans crainte et se méfiaient de leurs collègues, hommes comme femmes. C'est souvent à l'occasion des contacts que Djemila Zeneidi a essayé de nouer avec les ouvrières que celles-ci ont pris conscience qu'elles ne pouvaient la recevoir librement dans leur lieu d'habitation. Ce sont donc les tentatives d'accès de personnes extérieures à leurs logements qui leur ont permis de prendre la pleine mesure de la surveillance à laquelle elles étaient soumises. En dépit des contraintes matérielles de réalisation de l'enquête, en termes d'espace et de temps, cette démarche hors les murs a toutefois permis de recueillir une parole partiellement détachée du contrôle exercé par les employeurs et par la mairie.

22 Face à l'impossibilité d'entrer dans les labour camps par la voie hiérarchique, les enquêtes ont ici été rendues possibles par des contacts établis directement avec les personnes enquêtées à l'extérieur des camps. Cet accès au terrain a été autorisé par des frontières matérielles moins nettes et des régimes de confinement plus souples que ceux des centres de rétention et des prisons. Mais il a été fortement contraint par l'absence d'obligation à rendre public ce qui se passe entre leurs murs et le maintien à distance des regards extérieurs. Les recherches sur les camps de travailleur.ses révèlent ainsi un processus de compensation de la « détotalisation » des institutions de réclusion évoquée plus haut via la diffusion de dispositifs de surveillance plus discrets: nos enquêtes ont été conditionnées par le continuum socio-spatial de la contrainte.

\section{Déplacement de l'enquête : saisir l'enfermement comme continuum socio-spatial}

Si le continuum s'est révélé à travers l'étude de lieux de confinement difficilement accessibles aux chercheures, l'hypothèse de son existence a également poussé certain.e.s d'entre nous à élargir leurs investigations au-delà du lieu visé. L'objectif était alors d'adopter une focale non exclusivement axée sur le lieu d'enfermement en menant des entretiens à l'extérieur (de la prison, des logements contraints ou des 
centres de rétention). Cette démarche a favorisé l'ouverture de la problématique de départ ainsi que l'élargissement de l'éventail des acteur.trices interrogés (des surveillant.e.s et policier.e.s, mais aussi d'ancien.ne.s reclus.es, leurs proches, ou encore des responsables gouvernementaux ont pu être interviewés de cette manière). Elle a permis de reconstituer des parcours, judiciaires ou migratoires, dans lesquels la réclusion ne représente qu'une étape. Cette option méthodologique nous a par conséquent aidé.e.s à replacer le lieu clos dans l'espace social dans lequel il s'insère (et dont le projet institutionnel vise précisément à le couper), à saisir les continuités et porosités entre espaces d'enfermement et de liberté, mais aussi à recueillir une parole libérée de la promiscuité et de l'absence de confidentialité qui caractérisent les lieux fermés.

24 Ainsi, Olivier Clochard a complété son enquête sur la zone d'attente de Roissy en investiguant des lieux connexes : le tribunal de grande instance de Bobigny ${ }^{12}$ et la Cour administrative d'appel de Paris, dans lesquels il s'est rendu lors des audiences de jugement de migrant.e.s en instance d'expulsion; mais aussi les zones d'attente de province, dans lesquelles il a rencontré des policier.e.s ayant auparavant travaillé à Roissy. Il a également ouvert son enquête par des entretiens avec des officiers en exercice ou anciennement employés à ZAPI 3, tous rencontrés à l'extérieur des zones d'attente. Olivier Clochard a enfin rencontré des officiers de liaison ${ }^{13}$ en poste à l'étranger qui lui ont fourni des rapports statistiques sur les expulsions opérées depuis Paris, autant d'informations a priori «décentrées " mais éclairant avec précision le fonctionnement de la zone d'attente de Roissy.

L'enquête à la sortie du dispositif d'enfermement constitue une dernière option, retenue sur certains terrains. Elle présente l'avantage de permettre une étude longitudinale et d'assurer un suivi dans la durée des personnes interrogées, tout en déplaçant le regard sur les retombées de la réclusion à l'extérieur. Cette démarche fait émerger certaines facettes de l'enfermement parfois difficiles à saisir par l'entretien ou l'observation dans les lieux. Elle permet de croiser les récits tenus en situation de confinement et ceux livrés à l'extérieur. Les filtres qui s'exercent sur la production discursive varient en effet en fonction du lieu et du moment où le récit est recueilli. À l'extérieur, les récits peuvent être plus développés, répétés, donnant lieu à une reconstitution de l'expérience de l'enfermement que le temps écoulé depuis la sortie a par ailleurs permis de mettre partiellement à distance. Ainsi, le travail de bénévole de Bénédicte Michalon, en charge d'accompagner et d'organiser des activités de loisir à destination des ex-retenus au sein d'une ONG roumaine, s'est accompagné de l'autorisation à conduire des entretiens avec les personnes libérées de rétention et hébergées par l'ONG. Elle a signé avec l'ONG un contrat comportant une clause de confidentialité, mais la structure non gouvernementale lui a ensuite laissé une grande liberté d'initiative et ses activités de bénévolat ont finalement été assez distinctes de ses préoccupations de recherche, de telle sorte qu'elle a pu mener de front les deux démarches tout en respectant l'accord passé avec l'ONG. Sa présence quotidienne dans les locaux de l'ONG et l'intérêt manifesté envers les personnes qui y résidaient se sont traduits par la création de liens durables permettant de fréquentes entrevues, plus ou moins formalisées.

26 La conduite d'un suivi longitudinal est par ailleurs aujourd'hui favorisée par l'usage des technologies de l'information et de la communication (TIC). La possession d'un téléphone portable s'étant généralisée, il est désormais possible de maintenir des liens 
téléphoniques avec des individus dont la trajectoire ne s'arrête pas à la situation dans laquelle nous les avons rencontrés. Lorsque l'usage du téléphone portable était autorisé dans le lieu d'enfermement, certain.e.s d'entre nous sont parvenu.e.s à rester en relation avec des personnes recluses. L'utilisation des réseaux sociaux et de la téléphonie par internet, peu onéreuse, sont aussi des moyens de maintenir des contacts en dépit de la distance et du temps. Ainsi, l'enquête a pu continuer - ou parfois même commencer - hors du lieu clos, aussi bien pour Olivier Clochard (2014) à Chypre que pour Djemila Zeneidi en Espagne, après l'établissement de contacts et la prise de rendez-vous avec les saisonnières agricoles marocaines dont les numéros de téléphone avaient été obtenus lors des rencontres dans la rue. Le téléphone a également permis à certaines d'entre elles de contacter l'enquêtrice et d'improviser un rendez-vous lorsqu'elles ont pu se dégager du contrôle de l'employeur. Toutefois, au travail et dans les logements, l'usage du téléphone faisait l'objet d'une surveillance étroite exercée par le patron et relayée par certaines ouvrières. Ces dernières s'épiaient et écoutaient les conversations téléphoniques des unes et des autres pour en rendre compte à l'employeur, dans un contexte de compétition visant à obtenir les faveurs de celui-ci et s'assurer de la reconduite du contrat de travail. Le téléphone est donc à la fois un medium permettant sur certains terrains de compenser les problèmes d'accès aux enquêté.e.s, et un important vecteur de contrôle pour les institutions elles-mêmes

Quels que soient pour nous les garants de l'accès aux lieux fermés, agent.e.s de l'Etat, ONG ou employeur.se.s, et quel que soit le pays, les négociations, justifications et mises en scène dans lesquelles nous avons été impliqué.e.s pour pouvoir mener l'enquête ont pleinement participé de la recherche. Elles s'apparentaient à un parcours initiatique qui a influencé l'analyse. La nécessité de trouver des ajustements, voire des contournements ou de véritables solutions de repli hors les murs, nous a poussé.e.s à nous décentrer du seul lieu clos et à chercher à comprendre l'insertion de celui-ci dans un ensemble de dispositifs institutionnels de contrôle et d'assistance. Néanmoins, audelà des gains en termes de décentrement ou de décarcéralisation, ces choix multiples débouchent sur des solutions fragiles et nous ont obligé.e.s à sans cesse nous adapter à la contrainte, qui continue de s'exercer dans les lieux de l'enquête.

\section{Conclusion : une recherche sous contrainte}

Nous avons commencé nos investigations empiriques sur l'enfermement en souhaitant enquêter à l'intérieur même des établissements destinés à la détention, à la rétention administrative des étranger.e.s, ou à l'hébergement sous surveillance de certaines catégories de main-d'œuvre étrangère. Nous n'envisagions pas d'autres procédés que celui-ci pour recueillir le matériau empirique. La question de l'accès à ces lieux s'est donc immédiatement posée ; elle induisait automatiquement celle du temps passé sur place et de la capacité que nous aurions - ou non - à y rester suffisamment longtemps pour répondre aux besoins de l'enquête. Nous tirons aujourd'hui plusieurs conclusions de cette expérience collective, lesquelles sont à concevoir comme des résultats d'une réflexion méthodologique collective et aucunement comme des caractéristiques généralisables qui contribueraient à essentialiser ou à penser comme spécifiques les lieux d'enfermement. 
et à nous décentrer du seul lieu clos. Cette position, qui était au départ une position par défaut, s'est révélée donner davantage d'ampleur à nos démarches: elle nous a poussé.e.s à resituer les espaces d'enfermement dans des ensembles institutionnels et sociaux plus vastes, dans des temporalités et des spatialités débordant la période de réclusion. L'enquête in situ prend du relief lorsqu'elle va de pair avec des investigations hors les murs, tout particulièrement dans un contexte où la détotalisation d'institutions d'enfermement donne place à des surveillances plus diffuses, plus souples, moins perceptibles, à l'image de ces travailleuses marocaines prenant conscience de l'enfermement subi à la faveur d'une simple demande, formulée dans le cadre de la recherche, de leur rendre visite à leur lieu d'hébergement.

L'accès a par ailleurs pris une tournure différente en fonction des types de lieux. Contrairement à ce que nous imaginions initialement, les accréditations d'entrée dans les établissements les plus fermés et les plus sécurisés (prisons et centres de rétention) nous ont été accordées plus aisément que celles pour les logements contraints de travailleurs. Cet aspect du terrain a mis en lumière le fait que certaines institutions publiques, soumises à l'injonction à la transparence, sont inscrites depuis longtemps dans des débats publics et par conséquent plus ouvertes à la recherche que celles sous contrôle privé. Le développement de formes de privatisation de l'enfermement des étranger.e.s risque de fermer graduellement l'accès des chercheur.e.s à ces lieux.

Enfin, une part de l'enquête sur les lieux d'enfermement relève bien moins de l'autorisation ab initio que de la négociation toujours continuée : avec les autorités, mais aussi avec les ONG qui nous ont parfois donné la possibilité de travailler en leur sein en tant que bénévoles et, bien sûr, avec les personnes recluses. Craignant que les portes qu'ils ou elles ont réussi à ouvrir (ou entre-ouvrir) ne se referment, les chercheur.e.s se livrent aussi à différentes formes d'autocensure in situ. Au fil du temps passé dans des espaces contraints, nous avons pris conscience de notre intégration, plus ou moins fragile, des représentations dominantes du « danger » qui y ont cours (Le Caisne, 2007). Nous nous sommes aussi parfois limité.e.s dans nos déplacements, évitant par exemple des quartiers de détention ou les cellules d'isolement, sans même avoir eu à subir le contrôle des acteurs institutionnels. Face à un certain inconfort physique et moral, nous avons alors adopté des attitudes et habitudes qui, si elles nous ont permis de continuer nos investigations, ont également pu créer une sorte de stratégie de défense face à la violence qui traverse la réclusion et qu'il est difficile d'ignorer ou de mettre à distance. Par ailleurs, quel que soit le statut des chercheur.e.s, la relation d'enquête qu'ils ou elles tentent de construire est corrélée à l'émergence d'attentes particulièrement fortes chez les enquêté.e.s, et notamment les personnes enfermées. Dans cette perspective, le contexte de la recherche joue un rôle important: d'autres auteurs.rices ont déjà montré que le caractère "sensible » d'une enquête était, en partie, défini par la règle « du don/contre-don » structurant la relation enquêteur.rice/ enquêté.e (Bouillon, 2005). Ainsi, pénétrer dans un lieu d'enfermement implique de savoir poser les limites de cette relation afin de ne pas endosser un rôle qui ne pourrait par la suite être assumé («avocat» pouvant obtenir la libération du retenu, entremetteur capable d'organiser la vie dans l'illégalité en cas de non expulsion, etc.) (D'Halluin, 2005). Autrement dit, il s'agit de savoir jusqu'où s'engager sans risquer d'une part de briser la confiance des enquêté.e.s et de celles et ceux qui nous ont donné l'autorisation d'enquêter, d'autre part de perdre de vue les objectifs de production de connaissance qui motivaient originellement les investigations. Cet enjeu prenait un relief tout particulier dans notre enquête, où notre altérité de nationalité, de genre, de 
statut social était forte; et surtout, nous étions libres face à des personnes qui ne l'étaient pas: « l'accumulation de ces différences peut générer méfiance, voire hostilité. Mais une telle position présente aussi l'avantage pour son détenteur de ne pas devoir se justifier, se définir ni se situer à l'intérieur du groupe étudié, car il reste indiscutablement autre " (Massicard, 2002).

Bien que différant par certaines de leurs caractéristiques, les trois types de lieux traités par l'équipe entrainent des récurrences en termes de méthodologie d'enquête. La négociation conditionne l'enquête, puisque le moindre faux pas peut se traduire par une exclusion définitive du lieu. Elle la rythme, puisque la fréquence et la durée des incursions intramuros, et la place que le ou la chercheur.e y trouve, en dépendent. Les chercheur.e.s ne peuvent assurément pas s'extraire des rapports de pouvoir qui caractérisent les lieux de confinement. Toute la complexité de l'entreprise ethnographique réside alors dans la capacité des enquêteurs.rices à se faire une place en prenant en compte le caractère mouvant, fluctuant de ces rapports de pouvoir, perpétuellement recomposés et dans lesquels ils et elles se trouvent enserré.e.s.

\section{Bibliographie}

AGIER M. (2008), Gérer les indésirables. Des camps de réfugiés au gouvernement humanitaire, Paris, Flammarion.

AKOKA K., CLOCHARD O. (2015), « Régime de confinement et gestion des migrations sur l'île de Chypre », L'Espace politique, no. 25. En ligne : http:// espacepolitique.revues.org/3381.

BERNARDOT M. (2007), « Loger et déplacer les indésirables. Sociologie du logement contraint », in Levy-Vroelant C., Logement précaire en Europe. Aux marges du palais, Paris, L'Harmattan, pp. 286-303.

BOSWORTH M. (2017), « From the Field : Locking and Unlocking Detention », Blog Border Criminologies, University of Oxford. En ligne : https://www.law.ox.ac.uk/research-subject-groups/ centre-criminology/centreborder-criminologies/blog/2017/09/field-locking-and 2017

CHAMBOREDON H., PAVIS F., SURDEZ M., WILLEMEZ L. (1994), « S’imposer aux imposants. A propos de quelques obstacles rencontrés par des sociologues débutants dans la pratique et l'usage de l'entretien », Genèses, vol. 16, pp. 114-132.

CHANTRAINE G. (2004), Par-delà les murs, Trajectoires et expériences en maison d'arrêt, Paris, Presses Universitaires de France.

CHANTRAINE G., CHAUVENET A. (2005), « Le sociologue et la prison ou "la carcéralisation de la recherche" ", Intervention au colloque "Travailler sur la déviance. Problèmes méthodologiques et déontologiques des recherches en sciences sociales. Journée d'étude du réseau thématique « Normes, 
déviances et régulations sociales ", Association française de sociologie, Université Versailles SaintQuentin en Yvelines.

CHANTRAINE G., SCHEER D., MILHAUD O. (2012), « Espaces et surveillances en établissement pénitentiaire pour mineurs », Politix, vol. 1, no. 97, pp. 125-148.

CLIQUENNOIS G. (2006), « Problèmes méthodologiques inhérents à une recherche sociologique qualitative menée sur les politiques carcérales belges et françaises », Socio-logos. Revue de l'association française de sociologie, no. 1. En ligne : http://socio-logos.revues.org/28.

CLOCHARD O. (2014), «L'enfermement des étrangers à travers le prisme des papiers », Champ pénal/Penal field, vol. XI. En ligne : http://journals.openedition.org/champpenal/8858

CORBET A., MICHALON B. (2017), « Collaborer avec des organismes non gouvernementaux pour enquêter sur les camps de migrants et de déplacés. Le savoir comme enjeu professionnel », Migrations Sociétés, no. 167, pp. 53-62.

D'HALLUIN E. (2005), « Vaincre la suspicion, entrer dans une intimité douloureuse : une intenable extériorité », in Bouillon F., Fresia M., Tallio V., Terrains sensibles. Expériences actuelles de l'anthropologie, Paris, EHESS, pp. 55-74.

DARLEY M. (2008), Frontière, asile et détention des étrangers. Le contrôle étatique de l'immigration et son contournement en Autriche et en République tchèque, Thèse de doctorat en science politique, Institut d'Etudes Politiques de Paris.

DARLEY M. (2010), « Le pouvoir de la norme. La production du jugement et son contournement dans les lieux d'enfermement des étrangers », Déviance et Société, no. 2, pp. 229-239.

DARLEY M. (2012), «'Trouvez-vous une femme ici et tout s'arrangera...' L'intervention religieuse auprès d'étrangers placés en rétention ", Genre, sexualité et société, no. 8, pp. 1-18.

DARLEY M. (2014), « Le bon, la brute et le migrant ? Le rôle négocié des acteurs religieux dans un dispositif policier d'enfermement des étrangers en Allemagne », Sociologie du travail, no. 56, pp. 472-492.

DARLEY M., LANCELEVÉE C., MICHALON B. (2013), « Où sont les murs ? Penser l'enfermement en sciences sociales ", Cultures et Conflits, no. 90, pp. 7-20.

DARLEY M., LANCELEVÉE C. (2016), « Introduction. Faire tenir les murs. Pratiques professionnelles en milieu fermé », Sociétés contemporaines, vol. 3, no. 103, pp. 5-17.

EARLE R. (2014), «Insider and Out : Making Sense of a Prison Experience and a Research Experience », Qualitative Inquiry, vol. 20, no. 4, pp. 429-438.

FASSIN D. (2005), «L'innocence perdue de l'anthropologie : remarques sur les terrains sensibles », in Bouillon F., Fresia M., Tallio V., Terrains sensibles. Expériences actuelles de l'anthropologie, Paris, EHESS, pp. 133-146.

FASSIN D. (2015), L'ombre du monde. Une anthropologie de la condition carcérale, Paris, Le Seuil.

FERNANDEZ F., FISCHER N., KOBELINSKY C., MICHALON B. (2015), « Traiter, humaniser, judiciariser. Les transformations contemporaines de l'enfermement », Déviance et société, vol. 39, no. 4, pp. 371-377.

FISCHER N. (2007), La rétention administrative dans l'Etat de droit. Genèse et pratique du contrôle de l'enfermement des étrangers en instance d'éloignement du territoire dans la France contemporaine, Thèse de doctorat en science politique, Institut d'Etudes Politiques de Paris. 
FISCHER N. (2015), « De l'informalité à la "transparence”. Institutionnalisation et statut de la critique dans les réformes de la rétention administrative des étrangers en France ", Déviances et Société, vol. 39, no. 4, pp. 455-476.

FOUCAULT M. (1975), Surveiller et punir, Paris, Gallimard.

DE GALEMBERT C., HENNEGUELLE A., TOURAUT C. (2017) « Prison et méthode de recherche : présentation ", Criminocorpus, Prison et méthodes de recherche, Présentation de la journée d'étude. En ligne : http://journals.openedition.org/criminocorpus/3501.

GOFFMAN E. (1968 [1961]), Asiles. Etude sur la condition sociale des malades mentaux et autres reclus, Paris, Editions de Minuit.

HUGHES E.C. (1979 [1931]), The Chicago real estate board : the growth of an institution, New York, Arno Press.

JUAN S. (2006), «Le combat de l'Organisation et de l'Institution », SociologieS, Théories et recherches. En ligne : URL : http://sociologies.revues.org/582

KAMINSKI D., KOKOREFF M. (2004), «Ouverture », in D. Kaminski, M. Kokoreff, Sociologie pénale : système et expérience, Toulouse, ERES, pp. 7-13.

LAURENS S. (2007), « Pourquoi et comment poser les questions qui fâchent ? Réflexion sur les dilemmes récurrents que posent les entretiens avec les imposants », Genèses, no. 69, pp. 112-127.

LE CAISNE L. (2007), « De si dangereux condamnés. Construction sociale de la dangerosité en prison », Journal des anthropologues, no. 108-109, pp. 183-210.

LE CAISNE L. (2017), « De la confusion de l'ethnologue à la confusion des détenus », Criminocorpus, Prison et méthodes de recherche, Communications. En ligne : http://criminocorpus.revues.org/ 3527

LE CAISNE L., PROTEAU L. (2008), « La volonté de savoir sociologique à l'épreuve du terrain, De l'enchantement du commissariat au désenchantement de la prison », Sociétés contemporaines, vol. 72 , no. 4, pp. 125-149.

LIEBLING A. (2001), « Whose side are we on ? Theory, practice and allegiances in prison research ", British Journal of Criminology, vol. 41, no. 3, pp. 472-484.

MAKAREMI C. (2010), Zone d'attente pour personne en instance. Une ethnographie de la détention frontalière en France, Thèse de doctorat en anthropologie sociale et ethnologie, Université de Montréal.

MASSICARD E. (2002), « Être pris dans le mouvement. Savoir et engagement sur le terrain », Cultures \& Conflits, vol. 3, no. 47, pp. 117-143.

MICHALON B. (2015), «L'espace intérieur de la rétention. Policiers et retenus : travailler et habiter dans un lieu d'enfermement des étrangers en Roumanie ", Annales de géographie, no. 702-703, pp. 208-230.

MORAN D., PALLOT J., PIACENTINI L. (2009), « Lipstick, lace, and longing : constructions of femininity inside a Russian prison ", Environment and Planning D : Society and Space, vol. 27, no. 4, pp. 700-720.

OLIVIER DE SARDAN J-P. (1995), « La politique du terrain », Enquête, no. 1. En ligne : http:// journals.openedition.org/enquete/263

PALLOT J. (2007), « Gde muzh, tam zhena” (where the husband is, so is the wife) space and gender in post-Soviet patterns of penality ", Environment and Planning, no. 39, pp. 570-589. 
PIACENTINI L. (2004), Surviving Russian Prisons : Punishment, Politics and Economy in Transition, Cullompton, Willan Pub.

PIACENTINI L., PALLOT J., MORAN D. (2009), « Welcome to "Malaya Rodina” ("Little Homeland") : Gender and Penal Order in a Russian Penal Colony », Social \& Legal Studies, no. 18, pp. 523-542.

RHODES L. A. (2001), « Toward an Anthropology of Prisons », Annual Review of Anthropology, vol. 30, pp. 65-83.

SCHEER D. (2017), « Objets, espaces et corps du chercheur », Criminocorpus. Prison et méthodes de recherche, Communications. En ligne : http://criminocorpus.revues.org/3533

SCOTT J. (2009), Théorie critique de l'histoire. Identités, expériences, politiques, Paris, Fayard.

SNACKEN S. (2002), « “Normalisation” dans les prisons : concepts et défis. L'exemple de l'avantprojet de loi pénitentiaire belge », in De Schutter O., Kaminski D., L'institution du droit pénitentiaire, Bruxelles, Bruyant, pp. 133-152.

TURNBULL S. (2015), « Is it Ethical to Carry Keys for Research in Immigration Detention Centres? » Blog Border Criminologies, University of Oxford. En ligne : http:// bordercriminologies.law.ox.ac.uk/immigration-detention-keys/

TURNER J. (2016), The Prison Boundary : Between Society and Carceral Space, London, Palgrave Macmillan.

WACQUANT L. (2001), «Symbiose fatale », Actes de la recherche en sciences sociales, vol. 139, no. 2, pp. 31-52.

WACQUANT L. (2002), « The curious eclipse of prison ethnography in the age of mass incarceration », Ethnography, vol. 3, no. 4, pp. 371-397.

ZANNA O. (2010), « Un sociologue en prison », Nouvelle revue de psychosociologie, no. 9, pp. 149-162.

\section{NOTES}

1. Les dispositifs de l'enfermement. Approche territoriale du contrôle politique et social contemporain, coordonné par Bénédicte Michalon et financé par l'ANR (2009-2012) et le Conseil régional d'Aquitaine (2011-2015) (http://terrferme.hypotheses.org/). Cette recherche collective visait à appréhender le rôle de l'espace dans les rapports de pouvoir inhérents à l'enfermement et dans les expériences vécues par l'ensemble des acteur.e.s impliqués. Au-delà des contextes sociopolitiques et culturels propres à chaque société étudiée, nous cherchions à identifier des permanences, sans toutefois renier d'éventuelles ruptures et variations des processus d'enfermement.

2. Nous empruntons le terme de «logement contraint » à Marc Bernardot (2007). Nous utilisons en synonyme celui de camps de travailleurs, qui traduit l'appellation anglophone de labour camps.

3. L'enquête sur le logement des saisonnières agricoles marocaines en Espagne est mentionnée avec l'aimable autorisation de Djemila Zeneidi.

4. Les auteurs remercient Lucie Bony, André-Frédéric Hoyaux et Christophe Imbert pour leurs relectures des versions antérieures de ce texte ainsi que les deux évaluateurs anonymes pour leurs conseils avisés.

5. L'institution est entendue ici, dans une acception weberienne (reprise par Everett Hughes notamment, 1979 [1931]), comme un mécanisme de construction du pouvoir et de la prise de 
décision : groupement d'individus, l'institution contrôle différents aspects de leur vie et définit les statuts internes, mais aussi externes à son organisation (Juan, 2006).

6. Au Qatar, environ un million de migrant.e.s sont hébergé.e.s par leurs employeurs dans des labour camps. Leur liberté de mouvement hors de leur habitation est variable d'un camp à l'autre mais l'impression de cantonnement et d'enfermement est vivement ressentie par les travailleurs. 7. L'appellation renvoie nettement à l'univers guerrier et à l'idée qu'un prisonnier d'un Kosovo doit se battre pour sa survie. Il n'a toutefois pas été possible de retracer l'origine de cette néotoponymie sauf à faire le lien avec les événements européens.

8. La prison, construite en 1968 pour 1000 détenus, en abrite aujourd'hui entre 3000 et 4000 , répartis inégalement d'un quartier de détention à un autre.

9. Lieux de rétention où sont maintenu.e.s les étranger.e.s arrivant aux frontières et démuni.e.s de documents en règle.

10. ZAPI 3 (Zone d'Attente pour Personnes en Instance), avec une capacité officielle de 174 places, est la zone d'attente la plus importante en France.

11. La Cimade est une association qui entend défendre les droits des personnes migrantes et réfugiées. Voir notamment Fischer, 2007.

12. Après une période de maintien administratif de quatre jours, les étrangers enfermés en zone d'attente sont conduits devant le juge des libertés et de la détention afin que celui-ci décide ou non de prolonger la détention.

13. Selon le règlement du Conseil européen du 19 février 2004, un officier de liaison «Immigration » (ILO) est « un représentant d'un État membre détaché à l'étranger par le service de l'immigration ou par d'autres autorités compétentes [...] pour établir et entretenir des contacts avec les autorités [d'un ou plusieurs pays] en vue de contribuer à la prévention de l'immigration illégale et à la lutte contre ce phénomène, au retour des immigrés illégaux et à la gestion de l'immigration légale ».

\section{RÉSUMÉS}

Les recherches en sciences sociales sur l'enfermement reposent largement sur la conduite d'enquêtes qualitatives à l'intérieur des institutions de réclusion. Dans cette lignée, le texte livre une analyse collective de la méthodologie employée dans six contextes nationaux (Allemagne, Cameroun, Espagne, France, Qatar, Roumanie), par les membres de l'équipe TerrFerme au sein de lieux marqués par une fonction d'enfermement (prisons, centres de rétention administrative pour étranger.e.s, logements fermés pour main-d'œuvre étrangère). La thématique du degré d'ouverture et de fermeture de ces lieux, centrale dans leur traitement politique comme dans leur appréhension scientifique, s'est rapidement imposée comme le dénominateur commun de ces enquêtes et notamment comme enjeu méthodologique. Quelles ont été les différentes procédures mises en place par les chercheur.e.s pour accéder aux lieux visés ainsi que sur leurs effets sur la conduite des enquête? Ces cheminements méthodologiques se trouvent constitués en leviers de réflexion. Enquêter entre les murs est aujourd'hui possible grâce au mouvement d'ouverture relative des institutions de réclusion: les négociations menées avec les autorités publiques et avec des acteurs non gouvernementaux ont ouvert à certain.e.s d'entre nous les portes des lieux visés et ont largement conditionné nos positions intramuros. Cependant, les difficultés rencontrées par d'autres ont montré les limites de ce mouvement et nous ont 
poussé.e.s à enquêter également hors les murs : c'est la structuration de la surveillance en un continuum social et spatial qui a alors guidé nos enquêtes.

Social science research on confinement relies heavily on the conduct of qualitative surveys within confinement institutions. This text provides a collective methodological analysis based on the investigations carried out in six national contexts (Cameroon, France, Germany, Qatar, Romania, Spain) by the members of the TerrFerme research team. The investigations are carried out in confinement settings (prisons, administrative detention centres for foreigners, closed accommodation for foreign workers). The theme of the openness/closedness of these places, central to their political treatment as well as to their scientific apprehension, quickly emerged as the common denominator of these investigations. Doing fieldwork within the walls of closed settings is now possible thanks to the relative openness of carceral institutions: negotiations with public authorities and with non-governmental actors have opened to some of us the doors of the places we wanted to investigate in, and have conditioned our positions inside. But the difficulties others encountered have shown the limits of this openness and have pushed us to also investigate outside those places: in these cases, the organisation of surveillance in a social and spatial continuum guided our fieldwork.

\section{INDEX}

Thèmes : Carnets de terrain

Mots-clés : enfermement, contrainte, prison, centre de rétention, camp de travailleurs, enquête, expérience, réflexivité

Keywords : Confinement, Constraint, Prison, Detention Centre, Labour camp, fieldwork, experience, reflexivity

\section{AUTEURS}

\section{BÉNÉDICTE MICHALON}

Passages (UMR 5319), Chargée de recherche CNRS.

benedicte.michalon[at]cnrs.fr

\section{TRISTAN BRUSLÉ}

Centre d'Etudes Himalayennes (UPR 299), Chargé de recherche CNRS.

tristan.brusle[at]cnrs.fr

\section{OLIVIER CLOCHARD}

Migrinter (UMR 7301), Chargé de recherche CNRS.

olivier.clochard[at]univ-poitiers.fr

MATHILDE DARLEY

Cesdip (UMR 8183), Chargée de recherche CNRS.

mathilde.darley[at]gmail.com

\section{OLIVIER MILHAUD}

UR Médiations, Sorbonne Université, Maître de Conférences.

milhaud.olivier[at]gmail.com 


\section{MARIE MORELLE}

Prodig (UMR 8586), Univ. Paris 1, Maîtresse de Conférences.

marie.morelle[at]univ-paris1.fr 